\section{Digital Image Restoration Microscopy}

In biology and biomedicine, optical microscopy has rapidly progressed from the simple observation of static cellular and sub-cellular components to increasingly complex studies of the interrelationships of structure, function and local biochemical environment $\left(\mathrm{pH}, \mathrm{Ca}^{++}\right.$concentration, etc.). Low noise $\mathrm{CCD}$ cameras have been critical in this ongoing evolution. Originally used merely to capture two dimensional low light images (fluorescence, absorption or Raman), these cameras are now being used to generate a variety of multidimensional data. These other dimensions may include spectral information, temporal (time resolved) information and more recently, the third spatial dimension.

In this article we examine a newly commercialized technique, called digi tal image restoration microscopy, that utilizes a high performance CCD camera to generate quantitative images.

The principles involved are fairly simple. The CCD camera is used to record a series of two dimensional $(x, y)$ images at different depths $(z)$ in a fluorescently labelled sample. This is performed by sequentially moving the focal plane of the microscope. The resultant image "slices" are then mathematically combined to create a full three dimensional image. This can be displayed on the system monitor, either as slices, or as a 3-D rendering, which can be rotated for viewing from any angle.

Of course, when the microscope is focused on a particular plane in the object, the CCD camera is also receiving out of focus light from outside this plane. The trick to producing well focused final images is to deal with this out of focus light in some way.

In confocal laser scanning microscopes JCLSMS), out of plane light is simply eliminated by spatial filtering with a confocal pinhole. Such a system is optically inefficient because so much of the light is discarded. For this reason, samples must be treated with large concentrations of fluorophores.

In digital image restoration microscopy, a conventional lamp source is used for illumination, and all the fluorescent light from the sample is collected
Computer algorithms then "reassign" locations for out of focus photons to produce a sharp image. The algorithms are empirically fitted to the individual microscope's optical performance during a simple training phase. Basically, a fluorescent bead, smaller than the microscope's optical resolution, is placed on the sample stage. In focus and out of focus images are recorded so that the software learns how the microscope's optics transform this light. In normal imaging operation, this socalled point spread function is then used by the system to reassign all out of focus photons to their correct $x y z$ points of origin.

This technique has been known in the research community for over 15 years, being pioneed by groups at the University of Massachusetts and the University of California (San Francisco). The proven techniques and algorithms developed by these researchers have been licensed and developed into commercial instruments by Scanalytics (Billerica, MA), Applied Precision, Inc. (Mercer Island, WA), ONCOR Imaging Systems (San Diego, CA), Universal Imaging (West Chester, CA), and VayTeck, Inc. (Fairfield, IA), using Photometrics CCD cameras. Although their instruments differ in details of both hardware and software, they are similar in that each consists of a cooled CCD camera coupled to a research grade optical microscope. In all, the CED is interfaced to a powerful microprocessor, the sample is flat-field illuminated by use of a filament lamp and fiber bundle

How do these instruments compare to CLSMs? According to Carl Brown of Applied Precision, "For biologists involved with in vivo studies, this technique is much less disruptive than a CLSM." First lower levels of fluorescent indicators and labels are required, because of the high optical efficiency of the microscope. Also, the cells are not subject to the heating and photobleaching effects of a tightly focused laser beam. Even with low intensity illumination, the images can be accumulated and stored very rapidly (a few seconds). As a consequence, dynamic processes can be studied in 3-D over extended periods of time. The refocusing of the photons, referred to as exhaustive photo reassignment, or EPR ${ }^{\mathrm{TM}}$ by Scanalytics, takes from a few minutes to tens of minutes depending on the hardware platform and the size of the image.

In terms of spatial resolution, these instruments are capable of $0.25 \mu \mathrm{m}$

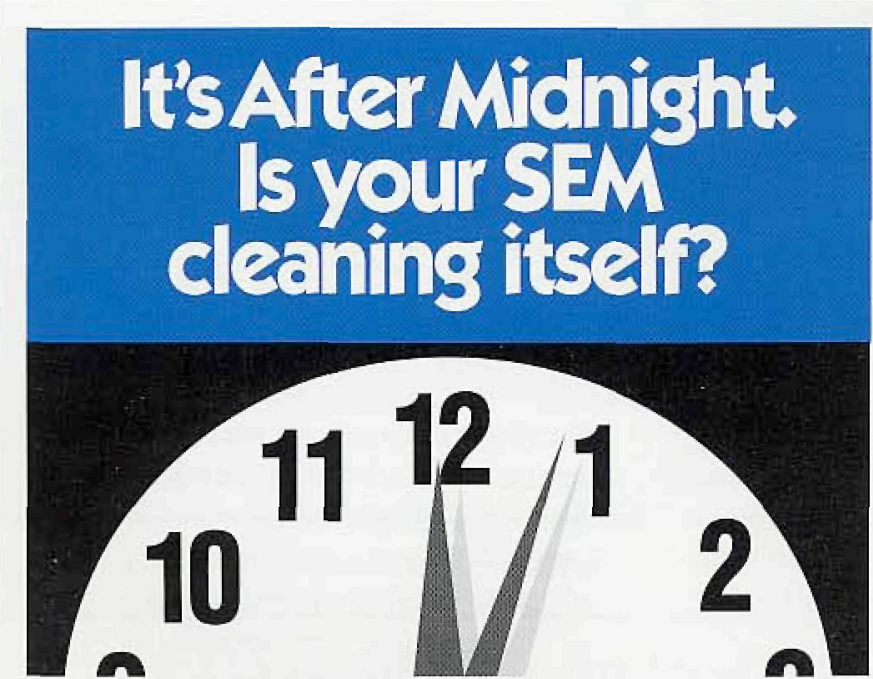

It would be if you had the SEM-CLEAN ${ }^{\text {TM }}$ anti-contamination system. Overnight, the inexpensive SEM-CLEAN system uses a Nitrogen purge to clean your electron microscope. Its active cleaning action removes oils and other adsorbed contaminants from the walls of the chamber and carries them to the roughing pump. Result? Significant reductions in, even elimination of, sample-contamination, raster burns and oil buildup on EDS windows. Over 50 satisfied SEM-CLEAN users are our best references. For a list and complete details, call or write today.

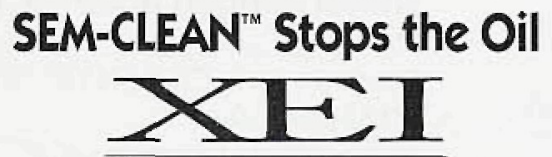

S C I E N T I F I C

3124 Wessex Way, Redwood City, CA, U.S.A 94061 415-369-0133 * Fax 415-363-1659

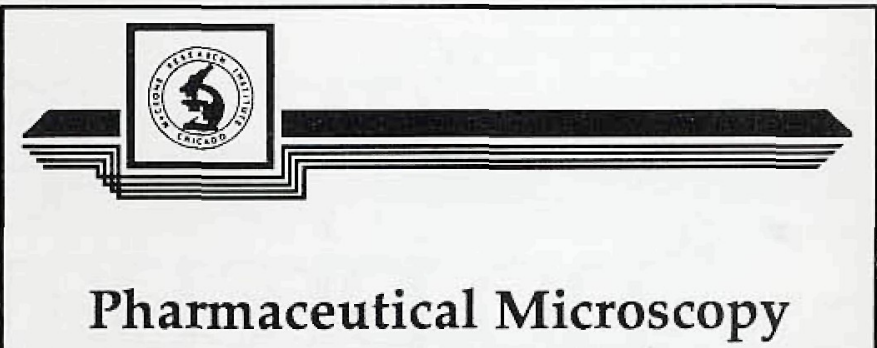

JANUARY 16-20, 1995 - HAYWARD, CA

February 13-17 and July 31-August 4-Chicaco, IL

McCrone Research Institute offers a course especially designed for the Pharmaceutical Scientist. This five-day course emphasizes the use of microscopy in the two major areas of interest to pharmaceutical researchers: identification of particulate contaminants in production quality control and parenteral solutions; and the influence of the solid state e.g., polymorphism, solvation and particle size on dosage formulation.

Call or write Nancy Daerr for detailed information about these and all microscopy courses.

\section{McCrone Research Institute}

\author{
2820 S. Michigan Avenue, Chicago, IL 60616-3292 \\ $312-842-7100$ - Fax 312-842-1078
}


resolution in the xy plane and $0.6 \mu \mathrm{m}$ resolution in the $z$ direction, which is certainly better than a CLSM even under optimal conditions. Summarizes Scanalytics' David Hitrys, "This technique allows us to breach what is typically thought of as the diffraction limit for light based microscopy."

So why has it taken so long for commercial products to emerge? Both companies are in agreement that it is the recent availability of CCD cameras combining low noise with high speed, together with affordable microcomputers with the power to run the "focusing" algorithms. States Hitrys, "Another benefit of our instrument is that the final reconstructed fluorescent image is quantitative, and can even be used for ratio imaging. In order to achieve this, a 12-bit dynamic range is an absolute minimum requirement."

Applied Precision's DeltaVision uses as Photometrics PXLTM with a Kodak (KAF 1400) CCD array. They chose $P X L^{T M}$ because its SCSI interface works directly with their SGI workstation. Adds Brown, "The KAF 1400 chip is virtually ideal because of its small, densely packed pixels, which translates directly into high spatial resolution." In terms of physical size, the KAF 1400 array also matches well with the central "flat field" portion of the microscope's image plane. Only this central region is used, because the refocusing algorithms are not quite as effective at the outside edges of the microscope's field of view.

To demonstrate the power of this technique, the following figure shows images of a NRK (normal rat kidney) containing a Rhodamine label for the protein tubulin. The left image is a typical $X Y$ slice recorded by the $C C D$, and the right image is after processing.

In conclusion, all companies predict that the combined benefit of 3-D information, high spatial resolution, and minimal in vivo disruption will lead to rapid growth of this technique, now that turnkey commercial instruments are readily available.

Reprinted from the Photometrics CCD newsbrief, Summer 1994. For additional information, contact Photometrics at telephone: (602)889-9933.

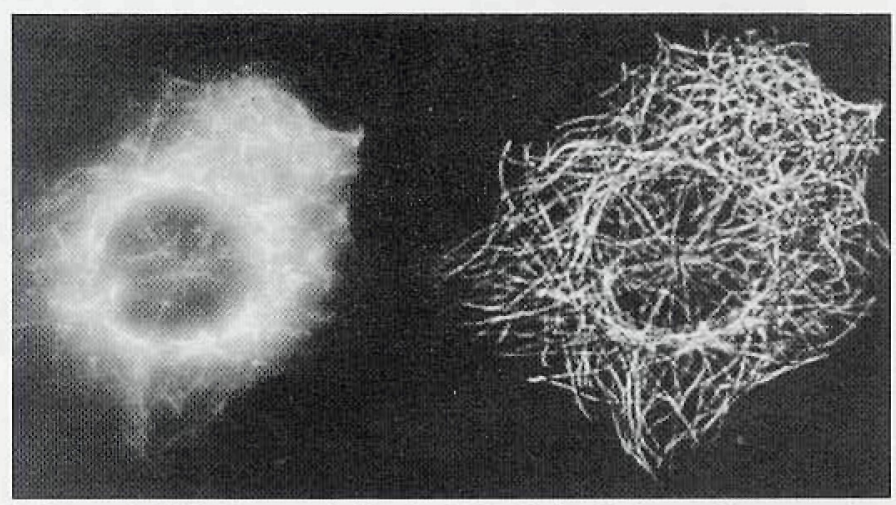

Left: a typical $X Y$ slice recorded by the CCD. Right: the image after processing. The entire image is only $71.4 \mu \mathrm{m}$ across. Image courtesy of Scanalytics

TRICKS OF THE TRADE
A common problem occurs for electron microscopists when the
area of interest in the sample is adjacent to a grid bar from the support
grid. If the micrograph taken includes the bar, it will show up on the
negative as a large clear space and will tend to fog the micrograph. This
problem can be overcome by adhering self-stick removable notes (e.g.,
Post-It-Notes) over the clear space on the non-emulsion side of the
negative. Then turn the negative over and trim away the excess paper
with a sharp razor blade. We use three pages stuck together to
completely block out the light.
Cynthia Goldsmith, Centers for Disease Control, Atlanta, GA.
-

\section{ROBINSON DETECTOR}

\section{WITH ANOTHER LEAP IN SEM IMAGING TECHNOLOGY ETP 辈咅 ONCE AGAIN LEADS THE WAY}

- RS-232 Communication.

- Intuitive Joystick Operation.

- Optimum Bandwidth Selection.

- True TV Imaging without Edge Smearing.

- Retractable Design for Maximum Flexibility.

- Computer Interface via Windows Now Available.

- 8 Line - 40 Character LCD Display of Detector Status.

- Instant Save and Recall of Operating Conditions.

- Microprocessor Control of the Robinson Detector.

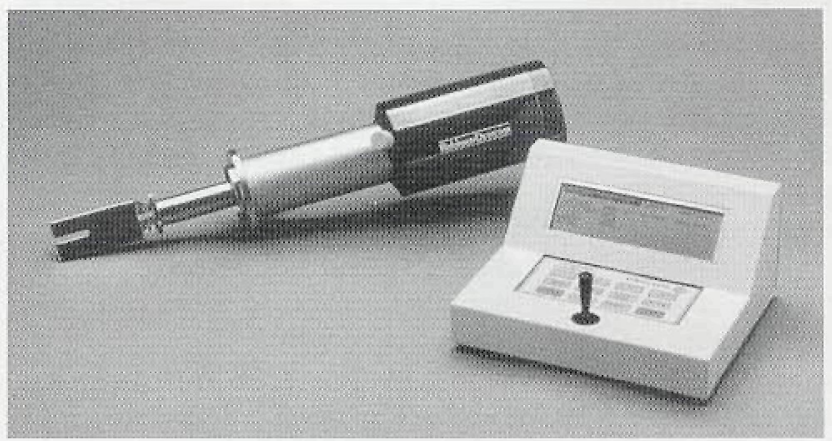

- Total Accessory Operation without using Valuable SEM Console Space.

- At Atomic Number 30, Discrimination is Better than 0.1 Atomic Numbers.

- $2000 \mathrm{~mm}$ Active Surface Area, $5 \mathrm{~mm}$ Thick at Beam Entry Point, 5:1 S/N at $1 \mathrm{PA}, 10 \mathrm{KV}$.

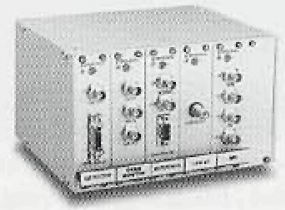

Extensive Accessory List including: Low Accelerating Voltage Operation, SE-BSE Signal Mixing, Selected Phase Imaging, Motorized Retraction, Scanning

Transmission, Cathodoluminescence Detection and Much More...

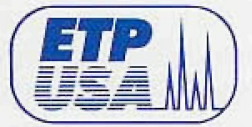

Electron Detectors, Inc. 1650 Holmes St. - Livermore, CA $94550 \bullet 1$ (800) 8ETP USA $\bullet$ (510) 449-8534 - FAX (510) 449-8996 